\title{
„Ohne Geld kann man ja nicht überleben oder nichts machen oder sich entfalten“
}

\section{Numerale Fähigkeiten von Erwachsenen mit Lernschwierigkeiten im Spannungsfeld der Grundbildung}

Wiebke Curdt ${ }^{1}$, Silke Schreiber-Barsch ${ }^{1,2}$

\author{
${ }^{1}$ Arbeitsbereich Lebenslanges Lernen, Fakultät für Erziehungswissenschaft, Universität Hamburg \\ ${ }^{2}$ Centre for Higher and Adult Education, Stellenbosch University, South Africa
}

\begin{abstract}
Zusammenfassung: Hintergrund: Auf Numeralität gerichtete Fähigkeiten von Erwachsenen sind ein wesentlicher Teil der Grundbildung Erwachsener als Prämisse und Ausgangsfundament für die Partizipation an Gesellschaft qua Lernen und Bildung. Forschungsbedarf besteht zu Numeralität von Erwachsenen mit Behinderung, insbesondere mit Lernschwierigkeiten, und zu einer Subjektperspektive auf Grundbildung. Methoden: Numerale Fähigkeiten von Erwachsenen mit Lernschwierigkeiten wurden aus der Beobachtung und Beschreibung von numeralen Praktiken rekonstruiert. Es ist eine Teilauswertung aus dem Datenkorpus eines Forschungsprojektes, das unter Prinzipien von Grounded Theory Methodologie und Partizipativer Forschung sowie in Anschluss an ein kulturwissenschaftliches Forschungsprogramm und Arbeiten der Disability Studies angelegt wurde. Ergebnisse: Der Umgang mit der numeralen Facette Größen \& Messen sowie deren Subkomponente der Größe Geld ist von hoher Relevanz für die Partizipation an Gesellschaft. Es belegt zugleich die existenten Fähigkeiten von Erwachsenen mit Lernschwierigkeiten für numerale Handlungen und Strategien im Umgang mit Geld. Diskussion: In dem rekonstruierten Umgang mit Geld spiegeln sich Schlüsselkontroversen der Grundbildung. Erkennbar wird das Potential einer ressourcenorientierten Grundbildung, die neben gesellschaftlicher Funktionalität auch den Eigen-Sinn und das Vermögen subjektgelagerter Strategien als Ressource der Gesellschaft und nicht als zu korrigierendes Defizit einzubringen vermag.
\end{abstract}

Schlüsselwörter: Numeralität, Mathematik, Behinderung, Lernschwierigkeiten, Grundbildung

"Without Money You Can't Survive or Do Anything or Develop Yourself": Numeracy-Related Abilities of Adults with Learning Difficulties in the Contested Terrain of Adult Basic Education

Abstract: Background: Adults' numeracy-related abilities constitute a significant part of adult basic education as premise as well as foundation of participation in society via learning and Bildung. The body of scholarship lacks research on numeracy and adults with disabilities, in particular with learning difficulties, and on a subject-oriented perspective on adult basic education. Methods: Numeracy-related abilities of adults with learning difficulties had been identified from data on observing and describing numeracy practices. This is derived from a partial analysis of the overall data from a research project, following principles of grounded theory methodology and participatory research as well as a cultural studies research paradigm and works by the disability studies. Results: Handling the numerate facet of size and measurements and in particular the size's subcomponent money shows to be of high significance for participation in society. It proves, in parallel, the existing abilities of adults with learning difficulties for successful activities and strategies with regard to handling money. Discussion: These findings bring nuance to the relevance of key controversies of adult basic education in order to further the quest for a resource-oriented adult basic education that acknowledges not only social functionality, but also individual sense-making and the potentiality of subject-related strategies as resources of society and not as a deficit to be fixed.

Keywords: Numeracy, mathematics, disability, learning difficulties, adult basic education

\section{Einleitung}

Die Frage nach der Grundbildung von Erwachsenen ist eng verbunden mit der Auseinandersetzung um Schlüssel- kompetenzen bzw. Schlüsselqualifikationen. Deren Identifikation und Entwicklung gehört seit den 1970er Jahren zu den erwachsenenpädagogischen Kernthemen; zugleich dokumentiert sich in ihnen der jeweils zugrunde gelegte 
„Verwertungskontext“ (Klein \& Alke, 2009, S.252). Erkennbar wird, welche konzeptionell „begründete(n) Sichtweisen auf die Menschen, ihre gesellschaftliche Verortung, Handlungsweisen und Handlungsintentionen" (Zeuner, 2009, S.262) zeithistorisch, gesellschaftlich und disziplinär angelegt werden, um Kompetenzen in bestimmten Inhalten und Qualitätsgraden - mit Grundbildung als Ausgangsfundament (Tenorth, 2004) - zu definieren.

Der Forschungsstand sieht Grundbildung als über die reine Wissensvermittlung von Techniken des Lesens, Schreibens und Rechnens hinausgehend an (z.B. Koller, 2020; Pape, 2018; Grotlüschen \& Buddeberg, 2020). Alltagsweltliche und anwendungsbezogene Kontexte jener Techniken sind genauso wie „Kompetenzen in den Grunddimensionen kultureller und gesellschaftlicher Teilhabe" (Bundesministerium für Bildung und Forschung \& Ständige Konferenz der Kultusminister der Länder in der Bundesrepublik Deutschland (BMBF \& KMK), 2012, S.1) einbezogen, die die „Grundlage für das Lernen und die Teilhabe an der Gesellschaft im Sinne des lebenslangen Lernens" (Europäische Kommission, 2018, S. C 189/4) gewährleisteten. Dieser Grundbildungs-PartizipationsNexus (Schreiber-Barsch \& Curdt, 2021) legitimiert Grundbildung als auch öffentliche Aufgabe (Nationale Dekade für Alphabetisierung und Grundbildung; 2016-2026): Grundbildung ist Prämisse wie Ausgangsfundament von Partizipation; unter der Annahme, dass Partizipation das individuelle Handlungsprinzip (Teilnahme an Lernen und Bildung) mit dem gesellschaftlichen Strukturprinzip (Teilhabe an Lernen und Bildung) (Beck, 2013) verschränkt. Demnach leiste Grundbildung einen zentralen Beitrag für Wissens- und Beschäftigungsanforderungen von Gesellschaften der Moderne; für Selbstbestimmung, Empowerment und kritisch-reflektierende Auseinandersetzung mit jenen Gesellschaften; genauso wie für die Reduktion sozialer Kosten bei einer nur gering literalisierten bzw. numeralisierten Bevölkerung (Craig, 2018, S.61-65; Gal, 2000; Grotlüschen \& Buddeberg, 2020; Rammstedt, 2013; Organisation for Economic Cooperation and Development (OECD), 2019; Yasukawa, Rogers, Jackson \& Street, 2018).

Jene alltagsweltlichen und anwendungsbezogenen Kontexte von Grundbildungsfähigkeiten stehen im Fokus der vorliegenden Studie. Sie untersucht den Teilbereich Numeralität in Bezug auf numerale Praktiken und zwar von Erwachsenen mit Lernschwierigkeiten (von anderen auch als sog. geistige Behinderung bezeichnet). Dies greift Kritik an quantitativ angelegten Kompetenzstudien mit der Zuweisung eines zu ,geringen Stellenwertes der Kontexte und der subjektiven Lebenswelten und daraus resultierenden Bedürfnissen" (Lüssenhop \& Kaiser, 2021, S. 47) und an der Fokussierung auf ein funktionales, objektiviertes Mathematikverständnis auf
(Schäfer, 2020, S.17-19), wonach bspw. im Rahmencurriculum Rechnen Teilnehmenden eine mathematische „Scheiternsbiografie“ (Meyerhöfer, 2017, S.5) zugeschrieben wird.

Demgegenüber begreift die hier diskutierte Studie mit ihrer kulturwissenschaftlich und handlungstheoretisch fundierten Ausrichtung Grundbildung als einen relationalen Terminus (Tröster, 2000, S.25) und als Teil eines Spannungsfeldes, in dem die Aushandlung pädagogischer Kontroversen um Inhalte, Klassifizierungsordnungen sowie Interessen und Machtverhältnisse analytisch einbezogen wird (Pape, 2018; Koller, 2020). Dies stärkt die pädagogische Perspektive auf Grundbildung (Grosche, Wohne \& Rüsseler, 2016), die die „Rolle des Subjekts als aktiv Lernenden“ betont, Professionelle als „Lernbegleitende" versteht sowie Lernerzentrierung, soziale Praxis, sinnhafte Erfahrungen und Interessen der Lernenden fokussiert (ebd., S.133-135). Die Befunde der Studie verweisen unter anderem auf die Bedeutung des Umgangs mit der Größe Geld als eine der inhaltlichen Facetten von Numeralität; die Rekonstruktion dessen bildet den Schwerpunkt des Beitrags.

\section{Forschungsbedarf: Numeralität und Behinderung}

$\mathrm{Zu}$ Numeralität besteht ein sich erst schrittweise ausdifferenzierender Forschungsstand; als vernachlässigt gelten, wie insgesamt in der Grundbildung, die Beforschung der Subjektperspektive (Tröster \& Schrader, 2016, S. 49; Werner, 2017, S. 42) genauso wie die der Subgruppe der Erwachsenen mit Behinderung (SchreiberBarsch, Curdt \& Gundlach, 2020; Curdt \& SchreiberBarsch, 2020) als eine der als vulnerabel angenommenen sozialen Gruppen. Es überwiegen Studien zu Mathematikunterricht (z.B. Fritz, Haase \& Räsänen, 2019), die, meist quantitativ angelegt, den Förderschwerpunkt geistige Entwicklung untersuchen (z.B. Lane, Herwegen \& Freeth, 2019; Garrote, Moser Opitz \& Ratz, 2015). Tan, Lambert, Padilla und Wieman (2019) kritisieren zudem in ihrer Metastudie über US-amerikanische Forschungsarbeiten (2006-2017) zu Schulmathematik und geistiger Behinderung die Dominanz kognitiver Testverfahren und eines behavioristischen Paradigmas. Insgesamt werde, so Ratz und Wittmann (2011, S.134), selten konzeptionell von einem aktiven Lernsubjekt ausgegangen und die Personengruppe nicht als grundsätzlich fähige, eigenständige "mathematics doers and thinkers" (Tan et al., 2019, S.1) anerkannt. Kompetenzstudien wie die PIAACStudie (Programme for the International Assessment of Adult Competencies) (Rammstedt 2013; OECD 2019) zur Messung alltagsmathematischer Kompetenz ver- 
zeichnet in ihrem Sample nahezu keine $(<0,4 \%)$ Personen mit einem Förderschulabschluss (Schreiber-Barsch et al., 2020, S.582-583).

\section{Relevanz der numeralen Umwelt}

Der Grundbildungs-Partizipations-Nexus weist Erwerb und Entfaltung von, hier: numeralen, Grundbildungsfähigkeiten mit der Intention ihrer Anwendung im Alltag (in Form von z.B. numeralen Praktiken) eine hohe partizipationseröffnende und/oder -ausübende Bedeutung zu. Diesbezüglich haben, in der Tradition eines kulturwissenschaftlichen Forschungsprogramms (Reckwitz, 2004), praxistheoretische Arbeiten zu situiertem Lernen (Lave 1988) und zu Numeralität als soziale Praxis (Street, Baker \& Tomlin, 2008; Yasukawa et al., 2018) die Relevanz der numeralen Umwelt (Evans, Yasukawa, Mallows \& Creese, 2017) hervorheben können. Evans u.a. (2017) definieren die numerale Umwelt der Subjekte als wechselseitiges Bedingungsgefüge aus, zum einen, Nachfrage und externen wie subjektiven Anforderungen (demands) und, zum anderen, den Komponenten des je spezifischen Angebotsspielraumes (affordances), die die (Nicht-)Performanz von Handlungen und Praktiken als Ausdruck der Konfiguration aus (nicht-)vorhandenen Gelegenheiten, Ressourcen und unterstützenden wie be-hindernden Faktoren beeinflussen. Ein Grundbildungskurs zu Rechnen kann, so Evans u.a. (2017, S.23), pädagogisch als unterstützende Maßnahme gedeutet werden - löst jedoch nicht automatisch, wie seit langem belegt, Nachfrage beim Subjekt aus (vgl. Bilger, 2016). Die je spezifische numerale Umwelt aus demands und affordances wirkt mithin entscheidend auf mehr bzw. weniger gelingende / $\mathrm{n}$ Erwerb und Entfaltung von Numeralität - als Prämisse wie Ausgangspunkt von Partizipation an Gesellschaft.

\section{Numerale Fähigkeiten als Reservoir der Subjekte}

Die Studie arbeitet mit einem weiten Begriff von Numeralität als "the knowledge and capabilities required to accommodate the mathematical demands of private and public life, and to participate in society as informed, reflective, and contributing citizens" (Geiger, Goose \& Forgasz, 2015, S. 531); er umfasst schul- $u$ nd alltagsmathematische Fähigkeiten (ebd., S.531). Schulmathematisch standardisierte Inhalte (Raum und Form; Zahlen und Rechnen; Größen und Messen; Muster und Strukturen; Wahrscheinlichkeiten, Häufigkeiten, Zufall; Konferenz der Kultusminister der Länder in der Bundesrepublik Deutschland (KMK), 2004, S.8) verschränken sich „ideengebend“ (Ratz \& Wittmann,
2011, Schäfer, 2020, S.67) mit auf einen Transfer gerichteten prozessbezogenen mathematischen Fähigkeiten (Problemlösen, Kommunizieren, Argumentieren, Modellieren sowie Darstellen; KMK 2004, S.7; Schäfer, 2020, S.71-75).

Für die Analyse des Umgangs mit der Größe Geld interessieren die Inhaltsebene (z. B. Geldscheine von Münzen unterscheiden, abzählen u.Ä.) (auch: Bitter, 2018) und, wie in der Mathematikdidaktik (für den Förderschwerpunkt geistige Entwicklung) festgehalten, insbesondere der Transfer in numerales Handeln als Ausdruck eines Sinnverstehens des Systems, „um für den Alltag aus dem Umgang mit Geld einen Nutzen ziehen zu können oder um wenigstens keinen Schaden zu nehmen" (Schmitz \& Scharlau, 1985, S.163). Dieser Transfer als (Nicht-)Anwendung von numeralen Grundbildungsfähigkeiten in numeralen Praktiken lässt einen analytischen Zugriff auf die „Rolle des Geldes in der Gesellschaft“ (Grassmann, 2013, S.10) und auf die „exponierte Stellung“, die „Geld (-Werte) hinsichtlich der unmittelbaren Bedeutsamkeit für die Lernenden [...] und ihrer späteren (selbständigen, selbstbestimmten) Lebenswirklichkeit“ (Schäfer, 2020, S.163) einnehmen, zu.

Numerale Fähigkeiten verstehen wir zunächst im Sinne von Fähigkeiten als „Kombinationen aus Handlungsdispositionen (handeln können)", die motorisches und kognitives Handeln sowie emotionales und motivationales Erleben inkorporieren, sowie „Wissen (wie zu handeln ist)“ (Straka \& Macke, 2009, S.15). Als Teil der finanziellen Grundbildung (Mania \& Tröster, 2018) beziehen wir numerale Fähigkeiten auf die „existentiell basalen und unmittelbar lebenspraktischen Anforderungen alltäglichen Handelns und der Lebensführung in geldlichen Angelegenheiten (ebd., 2018, S.13). Dies differenzieren Mania und Tröster (2015, S.23-24; Deutsches Institut für Erwachsenenbildung (DIE), 2019, S.15-17; DIE o.J.) anhand von sechs im Alltag situierten Kompetenzdomänen weiter aus: Einnahmen; Geld und Zahlungsverkehr; Ausgaben und Kaufen; Haushalten; Geld leihen und Schulden; Vorsorge und Versicherungen. Diese Domänen erfassen gleichwohl nur die Seite der (Handlungs-)Anforderungen bei alltäglichen finanziellen Angelegenheiten. Erwerb und Entfaltung von numeralen Fähigkeiten zielen - unter der Annahme des Nexus - auf einen ,kritische[n] Umgang mit Finanzen und Geld" (Lüssenhop \& Kaiser, 2021, S. 47) innerhalb machtdurchzogener gesellschaftlicher Verhältnisse und sollen ,einer zur Kritik befähigenden Bildung“ (ebd., S. 41) dienen.

Für die Untersuchung von numeralen Fähigkeiten unter Bedingungen von Behinderung folgen wir dem kulturellen Modell von Behinderung in Anschluss an Arbeiten der Disability Studies (z. B. Waldschmidt, 2017). Behinderung wird hier als ein Wechselspiel verstanden aus sozialen Strukturen und Praktiken, individuellen Haltungen, Be- 
einträchtigungen und körperlichen Wahrnehmungen sowie gesellschaftlich-kulturellen Normalitätserwartungen und Machtverhältnissen. Entsprechend verwenden wir den Begriff Menschen mit Lernschwierigkeiten, der von Selbstvertretungsorganisationen (People First) selbst gesetzt ist für Personen, die von anderen auch als geistig behindert oder beeinträchtigt bezeichnet werden (u.a. Bundesministerium für Arbeit und Soziales (BMAS), 2016, S.14). Jener Terminus hebt nicht auf individualisierte Beeinträchtigungen, sondern auf die Gemeinsamkeit kollektiver Lebenserfahrungen ab (Nieß, 2016, S.10). Weisser (2018) definiert demgemäß Fähigkeit als Beschreibung der basalen Einheit des „Vermögen[s], etwas zu realisieren" (Weisser, 2018, S.102). Jenes Vermögen ist als Reservoir, nicht zuletzt für Bildsamkeit als Resultat von Handlungsfähigkeit als anthropologischem Merkmal (Holm, 2018, S.112), in jedem Menschen angelegt (SchreiberBarsch et al., 2020, S. 584). Es entwickelt oder limitiert sich ,im Kontext von Erwartungen, Ressourcen und Gelegenheiten" (Weisser 2018, S.102) - bspw. denen einer numeralen Umwelt (Evans et al., 2017) und konkretisiert sich u.a. darin, im Umgang mit der Größe Geld im Bedingungsgefüge aus demands und affordances etwas handelnd (nicht) realisieren zu können bzw. zu wollen.

\section{Fragestellung}

Der Beitrag analysiert Befunde aus dem Datenmaterial eines Forschungsprojektes zu „Numeralen Praktiken von Menschen mit Lernschwierigkeiten" (NumPuD) (Schreiber-Barsch et al., 2020). Leitende Forschungsfragen des Projektes waren: Auf welche Weise wenden Menschen mit Lernschwierigkeiten numerale Praktiken im Alltag an? Welche numeralen Fähigkeiten spiegeln sich in den Praktiken? Und welche subjektiven Bedeutungen werden den Praktiken zugeschrieben? Forschungstheoretische und -methodologische Annahme war, dass numerales Handeln, Wahrnehmungen und Fähigkeiten, Interaktionen und Anwendungskontexte die materiale Ausdrucksgestalt sozialer Praxis abbilden. Diese Ausdrucksgestalt ist analytisch rekonstruierbar und lässt die Sinnstrukturiertheit menschlichen (numeralen) Handelns erkennbar werden - mit den diesem Handeln unterliegenden Fähigkeiten als Reservoir. Im vorliegenden Beitrag analysieren wir die im Datenmaterial rekonstruierten numeralen Fähigkeiten als Teil von numeralen Praktiken in Bezug auf den Umgang mit der Größe Geld; uns haben die Zuordnung der rekonstruierten Fähigkeiten zu den inhaltlichen Facetten von Numeralität sowie die jeweiligen Anpassungs- und Transferleistungen in ihrer Anwendung in Alltagssituationen über vier Lebensbereiche hinweg (Berufliche Bildung; Arbeit; Wohnen, Freizeit) interessiert.

\section{Methoden}

\section{Methodologische und methodische Rahmung}

Das Forschungsprojekt hat methodologisch Prinzipien Partizipativer Forschung (von Unger, 2014) mit der Grounded Theory Methodologie (GTM) in der handlungstheoretischen Tradition von Strauss und Corbin (1990) sowie Strübing (2014) verknüpft (ausführlich zur partizipativen Anlage des Projektes: Curdt \& Schreiber-Barsch, 2020; i.D). Untersuchungseinheit war die soziale Praxis (Williams et al., 2018), nicht individuelle kognitive Leistungsdispositionen bei numeralen Handlungen. Es wurden Grundprinzipien qualitativer Forschung (Offenheit, Fremdheit, Kommunikation/Kontextualität, Reflexivität; Mey \& Ruppel, 2018, S.209f), Leitprinzipien partizipativer Forschung (Doppelte Zielsetzung: Partizipation an Forschung und Partizipation an Gesellschaft; Gleichwertigkeit als Ziel; Umgang mit Wissen und mit Wissensproduktion; Macht und Kontrolle; Reflexion über die Rolle als Forschende; Curdt \& Schreiber-Barsch, i.D.; Curdt \& Schreiber-Barsch, 2020) sowie Leitprinzipien der gewählten GTM-Tradition ( $G e$ genstandsangemessenheit; empirische Sättigung der Theorie; theoretische Durchdringung; Praxisrelevanz; Prozesshaftigkeit; Interaktion; Strübing, 2014) befolgt. Als sensibilisierende Konzepte (Strübing, 2014, S. 29) des GTM-Prozesses fungierten neben der Systematisierung anhand von Lebensbereichen (s.u.) erstens die Numeralitäts-Definition gemäß Geiger et al. (2015) und zweitens der Forschungsstand zu den mathematischen Komponenten von Numeralität aus Bildungsstandards und Kompetenzstudien (KMK, 2004, S. 8), die wir zu einer Synthese der folgenden Facetten von Numeralität verdichtet und als heuristischen Analyserahmen genutzt haben: Raum und Form; Zahlen und Rechnen; Größen und Messen; Muster und Strukturen; Wahrscheinlichkeiten, Häufigkeiten, Zufall; sowie Artefakte (Schreiber-Barsch \& Curdt, 2021, S.208).

Methodisch haben wir die Rekonstruktion von numeralen Praktiken über in-situ Beobachtungen und subjektive Beschreibungen umgesetzt. Aus dem iterativen GTMProzess von Datenerhebung, -auswertung und Theoriegenerierung (theoretical sampling; Strauss \& Corbin, 1990, S.176-193) entwickelte sich folgendes Design:

Teilnehmende Begleitungen $(\mathrm{n}=11)$ (Bøe, Hognestad \& Waniganayake, 2017) mit Menschen mit Lernschwierigkeiten in deren Alltag: Numeralität beobachten (Beobachtungsprotokolle). Der explorative Zugang aus Subjektperspektive zielte auf die Erhebung und Rekonstruktion der Facetten von Numeralität in auch nicht-bewussten Anwendungsformen in Alltagssituationen (bspw. Routinen). (Der Begriff ,Beobachtung' konnte nicht verwendet werden, da dieser bei der von immer wiederkehrenden 
Diagnostiken und Tests sehr häufig belasteten Personengruppe direkt zur Verweigerung bzw. zum Abbruch der Erhebung geführt hätte [so wurden von dem Sample bspw. regelmäßig Rückversicherungen eingeholt, ob es sich tatsächlich nicht um einen „Mathetest" handeln würde]).

Teilstrukturierte Leitfadeninterviews $(\mathrm{n}=13)$ mit Menschen mit Lernschwierigkeiten: Numeralität beschreiben (Transkripte). Die Interviews sollten subjektive Beschreibungen, Bedeutungszuschreibungen sowie Handlungsmotive generieren. Als Erzählimpuls fungierte: Bitte erzähl/erzählen Sie uns etwas über Deinen/Ihren Alltag: Wie sieht Ihr/Dein Alltag aus? Im Folgenden wurden zudem Bildkarten zu Numeralität im Alltag im weitesten Sinne als Erzählimpulse genutzt; der Leitfaden sah Fragen zu den Themenfeldern numerales Lernen, Gelegenheitsstrukturen, Selbsteinschätzung und Wünsche vor.

Gruppendiskussionen $(\mathrm{n}=3)$ mit professionell Tätigen (gemäß Mangold, 1960): Numeralität als professionelle Aufgabe (Transkripte). Dies diente der kontrastiven Spiegelung der Beobachtungen und Beschreibungen sowie der Generierung von Aussagen über Potentiale und Grenzen der professionellen Unterstützung in Erwerb und Anwendung von Numeralität.

Das Design folgte forschungsethischen Standards (Dederich, 2017), bspw. zu einem informierten Einverständnis (Porter \& Lacey, 2005, S.92-95; Buchner, 2008, S.517) und zu Nutzen, Werten, Freiwilligkeit und Datenschutz (Dederich, 2017), unter insgesamt den Kriterien einer menschenrechtsbasierten, macht- und institutionenkritischen sowie verantwortungsbewussten Forschung mit Erwachsenen mit Lernschwierigkeiten (Schuppener, Heusner \& Müller, 2020). Die Auswertung aller erhobenen Daten folgte methodisch dem kategorialen Kodierverfahren der GTM-Tradition (s.u.).

\section{Sample}

Als Erwachsene galten Personen über 16 Jahre (gemäß bspw. Rammstedt, 2013). Das Sampling zielte auf eine konzeptionelle Repräsentativität (Strübing, 2014, S.31). Es wurde anhand der weithin als zentral ausgewiesenen Lebensbereiche von Menschen mit Lernschwierigkeiten systematisiert (gemäß BMAS, 2016, S. 20-22, 154; OECD, 2019, S.18): 1) Lernen und Bildung (Berufsbildung); 2) Arbeit bzw. Erwerbstätigkeit; 3) Wohnen und Lebenshaltung; sowie 4) Freizeit. Das Sampling nutzte das SchneeballVerfahren und erfolgte u.a. über Vorstellungen des Projektes $(n=11)$ an Orten der Lebensbereiche, an denen Erwachsene mit Lernschwierigkeiten üblicherweise anzutreffen sind. Voraussetzungen für eine Aufnahme in das Sample waren eine ausreichende Fähigkeit zu Kommunikation und eigenständige Mobilität.
Das Sampling folgte einem maximalen Kontrast mit Blick auf Alter und Lebenssituationen; einer Balance hinsichtlich von Gender und Lebensbereichen (ausführlich: Schreiber-Barsch et al., 2020, S.585-587; SchreiberBarsch \& Curdt, 2021, S.207):

Teilnehmende Begleitungen (TB): Zeitdauer: 2-7,5 Stunden. Alter: 18-80 Jahre. Gender: 6 männliche, 4 weibliche, 1 transgender Teilnehmende. Wohnsituation: 6 Teilnehmende lebten bei den Eltern, 3 in unterstütztem Wohnen, 2 lebten eigenständig. Lebenssituation: 2 Teilnehmende waren in Frühpensionierung, 4 waren angestellt, 4 besuchten berufliche Bildungsmaßnahmen, 1 absolvierte ein Praktikum.

Interviews (I): Zeitdauer: $30 \mathrm{~min}-2,5$ Stunden. Alter: 18-42 Jahre. Gender: 7 männliche, 6 weibliche Teilnehmende. Wohnsituation: 4 Teilnehmende lebten bei den Eltern, 4 in unterstütztem Wohnen, 5 lebten eigenständig. Lebenssituation: 7 waren angestellt, 3 in einer schulischen Maßnahme, 3 absolvierten ein Praktikum.

Gruppendiskussionen (GD) in den Lebensbereichen Berufliche Bildung, ambulant betreutes Wohnen und unterstützte Beschäftigung: Zeitdauer: 45-60 min. Gender: 8 weibliche, 7 männliche Teilnehmende. Fast ausschließlich pädagogische Berufsgruppen.

Drei Personen nahmen sowohl an einer Beobachtung als auch an einem Interview teil.

\section{Ergebnisse}

In der vergleichenden kategorialen Auswertung (offenes, axiales, selektives Kodieren; Strauss \& Corbin, 1996, S.43-117) des gesamten Datenmaterials zeigte sich die hohe Relevanz der Kategorie der sozialen Einbettung des numeralen Handelns neben der der subjektiven Bedeutungszuschreibung in der (Nicht-)Anwendung von numeralen Fähigkeiten (als Kontexte, Ursachen und Bedingungen in der Bedingungsmatrix; Strauss \& Corbin, 1996, S.133-134). Die Kategorie der sozialen Einbettung lässt Zusammenhänge von demands und affordances der jeweiligen numeralen Umwelt (Evans et al., 2017) deutlich werden; in der Anwendung treten im Datenmaterial vor allem die Facetten Größen \& Messen, Muster \& Strukturen sowie Raumorientierung als häufig beobachtete oder beschriebene Facetten hervor. Spezifisch der Umgang mit der Größe Geld - als Subkomponente von Größen \& Messen - tritt in alltäglichen Anforderungssituationen vielfach auf und wird mit hoher Relevanz belegt, wie folgende Sequenz aus einem Interview illustrieren kann:

Ich meine, das ermöglicht ja alles, was man hat, eine Wohnung, Essen, Kleidung, Freizeit. Das ist ja in unserer 
Zeit ... Ohne Geld kann man ja nicht überleben oder nichts machen oder sich entfalten. (I_1_\#01:00:19-1\#)

Es zeigt sich die thematisierte alltagsnahe und -weltliche Relevanz der Größe Geld als ein strukturgebender Bestandteil (Ratz \& Wittmann, 2011, S.146) von Kultur und Gesellschaft und für Selbstbestimmung und Partizipation. Dem entsprechen die sozialpolitischen Maßnahmen hin $\mathrm{zu}$ einem persönlichen Budget (BMAS, 2020) und einem Budget für Arbeit (Bundesgesetzblatt (BGBl), 2016). Neben Erwerb und Entfaltung von Fähigkeiten im Umgang mit Geld braucht es genauso eine numerale Umwelt, die den Umgang mit Geld einfordert und fördert (vgl. Abbott \& Marriott, 2012).

Entsprechend rekonstruieren wir im Folgenden die Anwendung von Numeralität in der Form von numeralen Praktiken im Umgang mit der Größe Geld. Entlang der Analysebegrifflichkeiten des GTM-Kodierparadigmas (Kontext, Ursachen, Bedingungen, Phänomene, Strategien (Strauß \& Corbin, 1996, S.133-134)) untersuchen wir die beobachteten und beschriebenen numeralen Praktiken: Ausgehend von den Kontexten (den Lebensbereichen: Berufliche Bildung, Erwerbstätigkeit, Wohnen und Freizeit) werden Ursachen für das Phänomen (Umgang mit der Größe Geld) erfasst sowie Bedingungen für Strategien der Subjekte. Aus den Phänomenen und den Strategien lassen sich numerale Fähigkeiten ablesen, die in der Tabelle 1 differenziert nach den Lebensbereichen, den numeralen Situationen der Anwendung (events; Street et al., 2008) und der zuzuordnenden numeralen Facetten systematisiert werden. Dies eröffnet Einblicke in numerale Handlungsfähigkeit als Wissen und Performanz, wie in alltäglichen Situationen und im Rahmen lebenspraktischer Anforderungen in der jeweiligen numeralen Umwelt Handlungen beschrieben bzw. beobachtet wurden. Hierzu verweisen wir auch auf die Arbeiten zu finanzieller Grundbildung (Mania \& Tröster, 2015, S.23-24; DIE, 2019; DIE, o. J.) sowie Mathematikdidaktik (Bitter, 2018; Schäfer, 2020). Alle Daten sind anonymisiert; eine Großschreibung in den Sequenzen verweist auf die Betonung des Gesagten.

\section{Ursachen und Kontexte}

Für die Summe an verfügbarem Geld sind zunächst Sozialleistungen bedeutsam. Damit verknüpft wiesen die unter der Kategorie soziale Einbettung rekonstruierten Ursachen (Subkategorie) für numerales Handeln im Umgang mit der Größe Geld dem Kontext (Subkategorie) des Lebensbereiches Arbeit eine hohe Bedeutung zu. Es greift die behinderungstypische Strukturierung in einen ersten und zweiten Arbeitsmarkt. Menschen mit Lernschwierigkeiten münden mehrheitlich in das System der Werkstätten für behinderte Menschen (WfbM) als dem zweiten Arbeitsmarkt (BMAS, 2016, S.119-124, 196, 222); auch wenn die Leistungen der Integrationsämter für die Arbeitsassistenz zur Integration auf dem ersten Arbeitsmarkt zwischen 2010-2014 auf 32\% stiegen. Als Regulierungen greifen v.a. die Sozialgesetzbücher IX und XII. Sie gewährleisten, insbesondere bei Lernschwierigkeiten, dass diese Personen in einem sozial für sie gesicherten System ihren Lebenslauf bestreiten können. 2019 verdienten Werkstattbeschäftigte bundesweit im Durchschnitt 206,95 €/Monat (Bundesarbeitsgemeinschaft Werkstätten für behinderte Menschen (BAG WfbM), 2020). Insgesamt geht Behinderung oft mit einer geringen sozioökonomischen Ausstattung und einem erhöhten Armutsrisiko (Trescher, 2018, S.144-145) einher. Änderungen scheinen seit 2018 mit dem Budget für Arbeit möglich.

\section{Bedingungen}

Bedingungen für Strategien im Umgang mit der Größe Geld kristallisierten sich unter der Kategorie soziale Einbettung in den Daten insbesondere in den direkten Interaktionen (Subkategorie) mit professionell Tätigen in allen Kontexten der Lebensbereiche heraus. Dies entspricht den im Forschungsstand belegten Bedingungen, wonach sich in Lebensbereichen und Tagesablauf von Menschen mit Lernschwierigkeiten häufig eine hohe Präsenz an und soziale Abhängigkeit von professionell Tätigen finden (Niediek, 2010; Nieß, 2016, S.10).

In der Beruflichen Bildung dokumentierte sich der Erwerb von Fähigkeiten für den Umgang mit Geld als zentral, so die Bedeutung von Geld als Tauschwert (Schmitz \& Scharlau, 1985, S.163) und die Berechnung von Geldbeträgen (Bitter, 2018, S.21):

Ich hab gerade einen Kurs "Rechnen im Alltag“ (lacht) mit den Schülern. [...] Und Umgang mit GELD halt, dass sie da so auch ein bisschen Bescheid wissen, was sie für ihr Geld bekommen können oder Rückgeld berechnen können für diejenigen, die schon fitter sind. (GD_1_B1_\#0O: 06:04-8\#)

In den Kontexten der Lebensbereiche Arbeit und Wohnen beschreiben und bewerten Professionelle den Umgang mit Geld, indem sie auf Herausforderungen wie bspw. die des digitalisierten Alltags mit spezifischen Kompetenzanforderungen zur Abwicklung von Geld und Zahlungsverkehr in Online-Shops sowie zum Haushalten als Grundlage von Kaufentscheidungen (DIE, o.J.) verweisen:

Ja, es; also es gibt wirklich Klienten, die KÖNNEN so einen Einkauf abschließen. Und sind auch in der Lage, 
ihre Bankverbindungen dort einzugeben. Und dann hagelt es auf einmal Mahnungen und das alles bläht sich auf von den Kosten, weil das KONTO nicht gedeckt ist. Dieser ZUSAMMENHANG ist nicht DA, ne? [...] Das Geld KOMMT ja immer. Und diese Geschichte jetzt so: Ich kaufe EIN, [...] Und ich hab aber überhaupt nichts aufder Bank. So, ich nehme dann die Ware AN und kann ÜBERHAUPT nicht verstehen, warum ich jetzt MAHNUNGEN kriege, warum das dann noch mal teurer wird, $n e$ ? (GD_2_B2_\#00:22:36-1\#)

Deutlich wird in der Sequenz zudem, dass aus professioneller Sicht mit Blick auf numerale Fähigkeiten weniger die Abwicklung, als vielmehr das Haushalten problematisiert wird.

Bedingungen zeigten sich in hohem Maße von der Situierung des numeralen Handelns (events) abhängig, so im Kontext Wohnen von der Wohnsituation. Ob eigenständig, ambulant betreut oder bei den Eltern wohnend beeinflusst das Vorhandensein von Gelegenheiten und Handlungsräumen für den Umgang mit Geld. Im Kontext Freizeit traten Bedingungen in Situationen von Freizeitaktivitäten und Konsum hervor, wie bspw. Shoppen, an einem Schwimm- oder Tanzkurs teilnehmen, seltener das Planen von Reisen oder Ausgaben für Kinder.

\section{Strategien}

Die Ursachen und Bedingungen rahmen das numerale Handeln der Subjekte in Bezug auf den Umgang mit Geld. Das Handeln lässt sich zu vier Strategien verdichten, die auf einer Dimensionalisierung zwischen zwei Polen als Spektrum möglichen Handelns verlaufen und zu denen sich das in den Daten rekonstruierte Handeln jeweils zuordnen lässt:

- Kontrollierendes, planendes Handeln <-> spontanes, konsumorientiertes Handeln;

- Handeln in Routinen <-> situationsgerechtes Handeln über Prioritätensetzungen.

In Situationen des Haushaltens (DIE, o.J.) zeigte sich im Datenmaterial neben dem Handeln in Routinen insbesondere die Strategie des kontrollierenden, planenden Handelns, wie die folgende Sequenz mit dem Abwägen von Kaufentscheidungen unter Berücksichtigung der Wünsche der Kinder illustriert:

Ja, wenn ich jetzt plane, auf den [Jahrmarkt] zu gehen, was das kostet, ne? Also man muss wissen, (Pause) Mittwoch ist Beispiel Familientag, da ist es günstiger auf dem [Jahrmarkt] als jetzt auf einen NORMALEN Tag. Aber ich gehe halt UNGERN auf den [Jahrmarkt], auch wenn meine Kinder das ... Ich mache das zwischendurch für meine Kinder halt, ne? Gehe ich dahin, aber sonst alleine würde ich da nicht hingehen oder so, ne? Aber da ACHTE ich halt auf die ... auch wieder MAL auf die Preise und SPARE mir aber ... Wenn jetzt meine Kinder sagen: Komm, lass uns dieses Mal auf den ... nächstes Jahr auf den [Jahrmarkt] gehen. Dann spare ich das vorher ab. [...] Ich muss mir das immer alles ZURECHTSPAREN. (I_2_\#00:17:03-5\#)

In Situationen des Einkaufens, die sich im Datenmaterial durchgängig finden, werden alle vier Strategien erkennbar; als Herausforderung tritt vor allem das Haushalten hervor. Mit der Strategie Kontrolle und Planen wird der Einkauf bezüglich der günstigsten Lebensmittel sondiert oder es werden Werbebroschüren gelesen, um den günstigsten Supermarkt vergleichend zu ermitteln. Es werden die Ausgaben in einem Haushaltsbuch notiert und am Wochenende überprüft, um zu wissen, was noch für den Rest des Monats übrig ist. Demgegenüber wird insbesondere von den Professionellen die Strategie des spontanen, konsumorientierten Handelns mit einer defizitorientierten Konnotation beschrieben, wonach bspw. im Kontext Wohnen einige bereits zu Beginn des Monats die Gelegenheit ergreifen und das (wenige) verfügbare Geld ausgeben würden:

Oh jetzt hab ich mich für die Zigaretten entschieden und hab nichts mehr zu essen, DOOF gelaufen. (lacht) Aber zwei Wochen später oder eine Woche später passiert so ziemlich das Gleiche wieder. ANDERSRUM: Ich hab einen Klienten, wenn der Anfang der Woche Geld kriegt, dann hatte er eingekauft, bis das weg war. Das musste SOFORT weg. (lachend) [ ...] Also irgendwie ist es immer VERDUNSTET. (GD_2_B2_\#00:36:22-O\#)

Die Strategie des Handelns in Routinen zeigt sich im Datenmaterial z. B. beim Einkaufen oder beim Haushalten. So wird bspw. immer eine bestimmte Summe abgehoben und/oder zum Einkaufen als Regulation der Ausgaben mitgenommen oder es werden immer bestimmte Produkte mit dem Wissen eingekauft, dass diese am günstigsten sind. Die folgende Einkaufssituation in einer TB zeigt in der subjektiven Anwendung der Größe Geld ein Handeln in Routinen als Ausdruck erworbener, kollektiv üblicher Handlungsmuster (Schmitz \& Scharlau, 1985, S.164): Tim platziert in einer bestimmten Reihenfolge die Ware auf dem Kassenband und zeigt numerale Fähigkeiten im System des Tausches von Geldwerten und Ware, überschlägt den Einkaufswert, bezahlt mit einer geringfügig höheren Münze und sorgt für die zu erfolgende Abrechnung mithilfe des Artefaktes (Kassenbon): 
Tim legt alle Sachen aufdas Kassenband. Dann ändert er die Reihenfolge „erst für die Leitung." Er nimmt die zwei Milchpackungen und legt diese nach vorn. „Das mache ich immer so."

Forscherin: „Legst Du das Geld aus?"

Tim: „Ja, bringe den Milch mit. Habe es so gelernt mit dem Bon. Bekomme meist dann $2 €$ oder 2,50 € von der Leitung zurück.“

Tim: „Schätze 1,58 €, um die 1,60 €, so, ... Pi mal Daumen“.

Die Kassiererin sagt, nachdem sie die Ware gescannt hat „1,56€, gut" und lacht.

Tim: „Das war ja schon ganz gut.“

Er nimmt zwei Euro aus seiner Schachtel und gibt sie der Kassiererin. Er bekommt das Geld zurück und den Bon. (TB_4, Tabelle 7, Nr. 1-6)

Schließlich reflektiert im Datenmaterial die Strategie eines situationsgerechten Handelns mit Prioritätensetzung nochmals stärker eigene Wünsche in Bezug zu vorhandenen Mitteln. Beispielsweise, beim Hobby des Gärtnerns sich teurere Pflanzen und Übertöpfe aus Geldgründen zu versagen und unter dieser Zielsetzung bewusst nur selten in den Baumarkt zu gehen:

Forscherin: „Wie oft fährst Du denn zum Baumarkt?“"

Tobias: „So selten wir möglich. Ich gebe dort immer viel Geld aus." (TB_11, Tabelle 7, Nr. 7)

\section{Numerale Fähigkeiten der Subjekte im Umgang mit der Größe Geld}

Aus den Beobachtungen und Beschreibungen lassen sich die rekonstruierten numeralen Fähigkeiten weiterführend systematisieren (im Abgleich zu DIE, o.J.; Bitter, 2018), indem diese anhand der Lebensbereiche, der numeralen Situationen der Anwendung (events) und der spezifischen numeralen Facette differenziert werden (siehe Tab. 1). Mit Artefakten sind Anschauungsmittel (z.B. Bilder, Listen, Rechnungen, Fahrpläne) sowie Arbeitsmittel (Technologien, Gegenstände, Objekte [z.B. örtliche Orientierungspunkte], menschliche Funktionsträger [z.B. Assistenz]) gemeint, die von den Subjekten eingesetzt und genutzt werden und die die Verfügbarkeiten ihrer numeralen Umwelt entscheidend bedingen.
Die soziale Einbettung und subjektive Bedeutung der events und der numeralen Fähigkeiten werden gleichwohl erst in der Kontextualisierung einer numeralen Praktik als Bedingungsgefüge aus Fähigkeiten, Handlungsmotiven, Gelegenheitsstrukturen und dem Verwertungskontext erkennbar. Die folgende Sequenz aus dem Lebensbereich Arbeit zeigt exemplarisch, wie die eigene Lebenssituation und daraus resultierende Partizipation an Gesellschaft nicht nur kritisch reflektiert, sondern mit Gestaltungswillen im Sinne einer ,zur Kritik befähigenden Bildung“ (Lüssenhop \& Kaiser, 2021, S. 41) unterlegt wird:

[Das hat mir] ... viel SPASS gemacht, das Autofahren. Da hab ich gesagt: Ich will was Berufliches machen. Und dann bin ich eben halt damals noch als Werkstatt-Mitarbeiter konnte ich dann diesen Beruf ausüben. Hab dann aber einfach mit der Zeit gemerkt, ich werde ganz schön ausgebeutet. Ich hab 40 Stunden gearbeitet und hab 220 Euro Lohn gekriegt. Werkstatt-Gehalt eben. Und der Auszubildende, der genau denselben Job gemacht hatte, der hatte, keine Ahnung, wie viel gekriegt. Ja, und dann bin ich da halt ein bisschen aufmüpfig gewesen und hab gesagt so: Ich mache hier genau denselben Job, 38 Stunden, und kriege hier echt so wenig Geld. Warum kann man nicht einen ausgelagerten Arbeitsplatz daraus machen, der zwar geschützt ist, aber wo ich AUCH wie im Rahmen der Unterstützten Beschäftigung so GELD verdienen kann. Haben sie nicht mit sich ma ... reden lassen. Klar, kostet ja Geld, ne? Ja, und dann bin ich da weggegangen, weil ich mich auch total unglücklich gefühlt hab, ausgenutzt, schlecht BEHANDELT, immer Ärger mit den Kollegen gehabt, mit den Vorgesetzten. (I_3 \#01:06:26-7\#)

\section{Diskussion}

\section{Grundbildung und Behinderung: Fähigkeiten im Spannungsfeld zwischen numeraler Umwelt und Subjekt}

Mit der empirischen Rekonstruktion von numeralen Fähigkeiten von Erwachsenen mit Lernschwierigkeiten und von Anpassungs- und Transferleistungen in ihrer Anwendung in Alltagssituation in Bezug auf den Umgang mit der Größe Geld konnte das vorliegende Datenmaterial einen Beitrag leisten, Befunde zum wirkmächtigen Spannungsfeld zwischen Gesellschaft und Subjekten, deren numeralen Handlungen und Fähigkeiten sowie unter Bedingungen von Behinderung weiter $\mathrm{zu}$ differenzieren und $\mathrm{zu}$ systematisieren. Jenes Spannungsfeld hatten Zeuner und 
Tabelle 1. Numerale Fähigkeiten von Erwachsenen mit Lernschwierigkeiten im Umgang mit der Größe Geld

\begin{tabular}{|c|c|c|c|c|c|}
\hline \multicolumn{6}{|c|}{ Numerale Fähigkeiten im Umgang mit der Größe Geld } \\
\hline Lebensbereiche & Numerale events & Zahlen \& Rechnen & Größen & Muster \& Strukturen & Artefakte \\
\hline $\begin{array}{l}\text { Berufliche } \\
\text { Bildung }\end{array}$ & $\begin{array}{l}\text { Unterricht; } \\
\text { Schulkiosk; } \\
\text { Mittagessen }\end{array}$ & $\begin{array}{l}\text { Zahlen nutzen } \\
\text { können; } \\
\text { seltener: Geld- } \\
\text { beträge addieren }\end{array}$ & $\begin{array}{l}\text { mit Geld (Münzen, } \\
\text { Scheinen) bezahlen }\end{array}$ & $\begin{array}{l}\text { Geldwertgefühl: reale } \\
\text { Preisvorstellungen } \\
\text { (bestimmte Produkte) }\end{array}$ & $\begin{array}{l}\text { Taschenrechner } \\
\text { Geld }\end{array}$ \\
\hline Arbeit & $\begin{array}{l}\text { Arbeit suchen; } \\
\text { Einnahmen; } \\
\text { Haushalten, } \\
\text { Beruf:Verkauf }\end{array}$ & $\begin{array}{l}\text { Zahlen/Geldwerte } \\
\text { (Münzen/Scheine) } \\
\text { unterscheiden, } \\
\text { abzählen und } \\
\text { nutzen können; } \\
\text { Geldbeträge } \\
\text { addieren } \\
\text { (im Verkauf) }\end{array}$ & $\begin{array}{l}\text { Vorstellung von Geld- } \\
\text { werten (Einheit: } € \text {, Cent); } \\
\text { Wissen um Einkommen / } \\
\text { Einnahmen (sehr genau } \\
\text { bis schätzen); } \\
\text { Preise (auswendig) } \\
\text { kennen; Geld wechseln, } \\
\text { herausgeben können } \\
\text { (Verkauf) }\end{array}$ & $\begin{array}{l}\text { Preise (Qualität) } \\
\text { vergleichen; } \\
\text { Geld als gegebenes } \\
\text { System verstehen } \\
\text { und nutzen können; } \\
\text { Zusammenhang/ } \\
\text { Vergleich: } \\
\text { Einkommen / Arbeits- } \\
\text { markt kennen (selten) } \\
\text { mit dem Wissen, } \\
\text { hohes, übliches, } \\
\text { geringes Einkommen }\end{array}$ & Geld \\
\hline Wohnen & $\begin{array}{l}\text { Haushalten, } \\
\text { Ausgaben } \\
\text { und Kaufen }\end{array}$ & $\begin{array}{l}\text { Zahlen/Geldwerte } \\
\text { (Münzen/Scheine) } \\
\text { unterscheiden, } \\
\text { abzählen und } \\
\text { nutzen können; } \\
\text { Haushalten: } \\
\text { Geld (ein-)teilen; } \\
\text { Geld zählen; }\end{array}$ & $\begin{array}{l}\text { Einkommen: Gehalt } \\
\text { (Tag des Erhalts } \\
\text { kennen); Geld über } \\
\text { eine bestimmte Zeit } \\
\text { (Monat) einteilen } \\
\text { können; } \\
\text { Ausgaben/Kaufen: } \\
\text { Einkaufssituation: } \\
\text { Vorbereitung, mit } \\
\text { (Münzen), Scheinen } \\
\text { (bestimmte Summe) } \\
\text { bezahlen; } \\
\text { selten: Münzen genau } \\
\text { abzählen; Preise } \\
\text { vergleichen, (ein-)- } \\
\text { schätzen; überschlagen } \\
\text { des Einkaufsbetrags; } \\
\text { Wissen, was etwas in } \\
\text { etwa kostet; ist günstiger }\end{array}$ & $\begin{array}{l}\text { Einnahmen: } \\
\text { Sozialleistungen/ } \\
\text { Einkommen kennen, } \\
\text { Ausgaben/Kaufen: } \\
\text { Ausgaben für } \\
\text { Wohnung verwalten; } \\
\text { routinemäßig Einkaufen } \\
\text { gehen (bestimmte } \\
\text { Produkte nehmen); } \\
\text { Preise vergleichen } \\
\text { Haushalten: sparen; } \\
\text { planen }\end{array}$ & $\begin{array}{l}\text { Geld, } \\
\text { Haushaltsbuch, } \\
\text { Geldkassette, } \\
\text { Einkaufszettel, } \\
\text { Preisschild, } \\
\text { Taschenrechner, } \\
\text { Kassenbon, } \\
\text { Bankkonto, } \\
\text { Bankkarte, } \\
\text { Werbezettel }\end{array}$ \\
\hline Freizeit & $\begin{array}{l}\text { Haushalten; } \\
\text { Ausgaben \& } \\
\text { Kaufen: } \\
\text { Shoppen, } \\
\text { Reisen } \\
\text { Sport treiben; } \\
\text { Zahlungsverkehr }\end{array}$ & $\begin{array}{l}\text { Zahlungsverkehr: } \\
\text { Zahlen/Nummer } \\
\text { (Bankkarte) } \\
\text { kennen/nutzen; } \\
\text { Ausgaben/Kaufen: } \\
\text { selten: mit Bank- } \\
\text { karte bezahlen; } \\
\text { Geldbeträge } \\
\text { addieren, Preise } \\
\text { lesen, verstehen, } \\
\text { einordnen können }\end{array}$ & $\begin{array}{l}\text { Haushalten: } \\
\text { mit Taschengeld } \\
\text { umgehen; } \\
\text { Ausgaben / Kaufen: } \\
\text { Wissen, was die Ware } \\
\text { in etwa kostet; über- } \\
\text { schlagen der Kosten; } \\
\text { Geld ausgeben }\end{array}$ & $\begin{array}{l}\text { Haushalten: } \\
\text { Einkommen-Kosten } \\
\text { der Ware vergleichen; } \\
\text { Wissen um Zusammen- } \\
\text { hänge (wenig Geld heißt } \\
\text { sich wenig leisten } \\
\text { können); Ausgaben/ } \\
\text { Kaufen: Preise } \\
\text { beachten, vergleichen }\end{array}$ & $\begin{array}{l}\text { Geld, Preisschilder, } \\
\text { Bankkarte (PIN) }\end{array}$ \\
\hline
\end{tabular}

Pabst (2011) bereits mit Blick auf Literalität und Erwachsene thematisiert; es äußere sich in „Diskrepanzen zwischen öffentlichen Erwartungen an Grundbildungsfähigkeiten, deren Fehlen zumeist mit Defizitzuweisungen verknüpft werden" (wie illustriert im Rahmencurriculum Rechnen; siehe Einleitung) genauso wie in ,subjektiven Begründungen literaler Praktiken, die zwar gesellschaftlich als nicht ausreichend angesehen werden, individuell aber durchaus adäquaten Strategien folgen" (ebd., S.34). Unter dem Blickwinkel der Kategorie Behinderung verschärft sich unseres Erachtens das Spannungsfeld angesichts von „Fähigkeitskonflikten“ (Weisser, 2018, S.100) aus „,ähigkeitsbezogenen Erwartungsverletzungen" (ebd.) in der Interaktion zwischen Subjekt und numeraler Umwelt, wie mit der o.g. Sequenz zur Entlohnung auf dem behinderungsspezifisch strukturierten Arbeitsmarkt illustriert. Fähigkeits- 
konflikte und Zuschreibungen von Behinderung werden derart in ihrer Verschränkung deutlich und damit diskutierbar; Grundbildung erhält eine politische Dimension unter dem Ziel möglichst selbstbestimmter Partizipation qua Lernen und Bildung.

Der eingesetzte forschungstheoretische und -methodologische Zugriff kann insofern Befunde erweitern, als dass mit dem genutzten Fähigkeitsbegriff eine ressourcen-, nicht defizitorientierte Sicht aus der Subjektperspektive in den Vordergrund der Grundbildungsdiskussion und der gängige Kompetenzbegriff mit dem Konzept gesellschaftlich-funktionaler Erwartungs- und empirisch messbarer Rangordnungen und dem Fokus auf v. a. kognitive Dispositionen (Klieme \& Leutner, 2006, S.879-880; Tan et al. 2019) in den Hintergrund tritt. Die subjektiv als sinnvoll und gelungen wahrgenommene Anwendung numeraler Fähigkeiten im Umgang mit der Größe Geld verweist auf die Notwendigkeit von demands und affordances in der numeralen Umwelt, um „Lernen als Akt der Selbstbestimmung" (Dinkelaker, 2018, S.64) nicht nur pädagogischprofessionell zu befördern, sondern auch aus Subjektsicht als Impuls und Motiv für Lernhandlungen wahrzunehmen und anzunehmen. Aus so einem Verständnis heraus wird es mithin, so eine Sequenz aus einer Gruppendiskussion mit professionell Tätigen, „HÖCHSTspannend diesen Logiken [der Menschen mit Lernschwierigkeiten bei der Anwendung von Numeralität; Anm. Autorinnen] nachzugehen" (GD_3_B3_\#00:54:44-6\#).

\section{Limitationen}

Das Forschungsdesign erlaubt keinen generalisierbaren Nachweis von Fähigkeiten für Erwachsenen mit Lernschwierigkeiten; vielmehr sollte das Spektrum der in den Daten empirisch rekonstruierten Fähigkeiten eines bestimmten Samples aufgezeigt werden, um Ansatzpunkte für weitere Forschung als auch für Fragen der Praxisrelevanz nach Optionen pädagogischer Unterstützung und Vermittlung im Umgang mit der Größe Geld zu liefern.

Dies führt zu der Frage, ob die Kritik an einem nur kaum vorfindbaren Einbezug der Personengruppe in Kompetenzstudien tatsächlich in eine Forderung nach ihrem Einbezug und einer Zuordnung zu Kompetenzstufen resultieren sollte - oder vielmehr Nutzen und Schaden solcher Forschung abzuwägen sind (Dederich, 2017). Es ist zu befürchten und forschungsethisch sowie mit Blick auf die genannten Gütekriterien und Leitprinzipien (s.o.) als problematisch einzuordnen, dass erneut eine Position als passiver Respondent gegenüber standardisierten Anforderungskatalogen zugewiesen und defizitorientierte Fähigkeitszuschreibungen reproduziert würden; hier stellt sich ein zentrales Forschungsdesiderat.

\section{Relevanz für die Praxis}

Unter der Annahme von Fähigkeiten als einem kontextuell eingebundenen Reservoir der Subjekte eröffnen sich Möglichkeitsräume professionell-pädagogischen Handelns in der Grundbildung unter dem Ziel von Partizipation. Das Spannungsfeld zwischen Subjekt und numeraler Umwelt und den Verwertungskontexten von Grundbildung zwischen Adaption an Gesellschaften der Moderne, Erweiterung subjektiver Handlungsfähigkeit und emanzipatorischem Gestaltungswillen bleibt jedoch grundsätzlich erhalten; das Subjekt kann nicht losgelöst von Kontext, gesellschaftlichen Bedingungen und personalen Gelegenheitsstrukturen teilhaben und teilnehmen.

Die Befunde verweisen auf die Notwendigkeit von lebensweltorientierten Kursangeboten und Curricula, die subjektiv bedeutsame Lernwege und -formen anerkennen und konzeptionell einbinden (Werner, 2017, S.23). Für Numeralität liegen auf die schulmathematischen Komponenten fokussierte Curricula für die Schule und für erwachsene Lernende vor (Deutscher Volkshochschulverband (DVV, 2017)), letztere gleichwohl deutlich weniger differenziert und meist auf Einzelkomponenten wie Finanzielle Grundbildung (DIE, 2019) begrenzt. Es fehlen „konzeptionelle Aussagen zur Berücksichtigung von Lernwegen jenseits von Bildungsstandards" und „außerhalb curricularer Vorgaben“ (Werner, 2017, S.23), d.h. auch außerhalb institutionalisierter Lehr-Lernsettings wie non-formale Grundbildungs-/Alphabetisierungskurse, und unter Einbezug emanzipatorischer und zu Kritik befähigender Bildung zur Entfaltung von Selbst-, Mitbestimmungs- und Solidaritätsfähigkeit (Klafki, 1991) entlang des Lebenslaufs. Mit Grosche et al. (2016, S.140) ist hier die Verschränkung von pädagogischen, soziologischen und psychologischen Perspektiven für einen multiperspektivischen und interdisziplinären Zugriff auf Grundbildung zu fordern und die Beachtung einer alltagsweltlichen Anwendung von Numeralität hin zu einem autonomen Modell von Numeralitäten (Street et al., 2008) zu befördern.

Mit Fähigkeiten als Reservoir und als „Vermögen, etwas zu realisieren“ (Weisser, 2018) können die „mathematics doers and thinkers" (Tan et al., 2019, S.1) zeigen, dass sie „[w]issen (wie zu handeln ist)“ (Straka \& Macke, 2009, S.15) im Wechselspiel mit sozialen Strukturen und Praktiken, gesellschaftlich-kulturellen Normalitätserwartungen und Machtverhältnissen. Diese Fähigkeiten sind in der Grundbildung aufzudecken, zu verstehen und zu unterstützen, um den Eigen-Sinn und die Potenzialität von subjektgelagerten Strategien als Ressource der Gesellschaft, nicht als zu korrigierendes Defizit einzubringen. 


\section{Literatur}

Abbott, D. \& Marriott, A. (2012). Money, finance and the personalisation agenda for people with learning disabilities in the UK: some emerging issues. British Journal of Learning Disabilities, 41, 106-113.

BAG WfbM - Bundesarbeitsgemeinschaft Werkstätten für behinderte Menschen e.V. (2020). Durchschnittliche monatliche Arbeitsentgelte 2017-2019 in Euro. https://www.bagwfbm.de/ file/1356 [29.06.2021].

Beck, I. (2013). Partizipation-Aspekte der Begründung und Umsetzung im Feld von Behinderung. Teilhabe, 52(1), 4-11.

Bilger, F. (2016). Kursforschung am Beispiel AlphaPanel. In C. Löffler \& J. Korfkamp (Hrsg.), Handbuch zur Alphabetisierung und Grundbildung Erwachsener (S.165 - 188). Münster: Waxmann.

Bitter, D. (2018). Der Größenbereich Geld - Rechnen mit Euro und Cent. Lernen konkret, 37(4), 16 - 21.

Bøe, M., Hognestad, K. \& Waniganayake, M. (2017). Qualitative shadowing as a research methodology for exploring early childhood leadership in practice. Educational Management Administration \& Leadership, 45(4), 605-620.

Buchner, T. (2008). Das qualitative Interview mit Menschen mit so genannter geistiger Behinderung - Ethische, methodologische und praktische Aspekte. In G. Biewer, M. Luciak, \& M. Schwinge (Hrsg.), Begegnung und Differenz: Menschen-Länder-Kulturen (S. 516 - 528). Klinkhardt, Bad Heilbrunn: Beiträge zur Heil- und Sonderpädagogik.

Bundesgesetzblatt (BGBI) (2016). Gesetz zur Stärkung der Teilhabe und Selbstbestimmung von Menschen mit Behinderungen. Bundesteilhabegesetz (BTHG). Auszug aus dem BGBI 2016 Teil I Nr. 66, vom 29. Dezember 2016. https://www.bmas.de/Shared Docs/Downloads/DE/Gesetze/bthg.pdf?__blob=publication File\&v=1 [03.03.2021].

Bundesministerium für Arbeit und Soziales (BMAS) (2016). Zweiter Teilhabebericht der Bundesregierung über die Lebenslagen von Menschen mit Beeinträchtigungen. Bonn: BMAS.

Bundesministerium für Arbeit und Soziales (BMAS) (2020). Das persönliche Budget. Bonn: BMAS.

Bundesministerium für Bildung und Forschung (BMBF) \& Ständige Konferenz der Kultusminister der Länder in der Bundesrepublik Deutschland (KMK) (2012). Vereinbarung über eine gemeinsame nationale Strategie für Alphabetisierung und Grundbildung Erwachsener in Deutschland 2012 -2016. Berlin.

Craig, J. (2018). The promises of numeracy. Educational Studies in Mathematics, 99(1), 57 -71. https://doi.org/10.1007/s10649018-9824-5.

Curdt, W., \& Schreiber-Barsch, S. (im Druck). Zur Heuristik einer partizipativ-qualitativen Erwachsenenbildungsforschung unter Anwendung der Grounded Theory Methodologie. Überlegungen am Beispiel von Erwachsenenbildung und Behinderung. In J. Engel, A. Epp, J. Lipkina, S. Schinkel, H. Terhart \& A. Wischmann (Hrsg.), Bildung im gesellschaftlichen Wandel - Qualitative Forschungszugänge und Methodenkritik. Schriftenreihe der DGfE-Kommission Qualitative Bildungs- und Biographieforschung. Leverkusen: Verlag Barbara Budrich.

Curdt, W., \& Schreiber-Barsch, S. (2020). Abilities in the blind spot of testing regimes: Eliciting the benefits and limitations of participatory research approaches on numeracy in adult basic education. International Review of Education, 66(2-3), $387-413$.

Dederich, M. (2017). Ethische Aspekte der Forschung an Menschen mit geistiger Behinderung. Teilhabe, 56(1), S.4-10.

Deutscher Volkshochschulverband (2017). Rechnen. DVV-Rahmencurriculum (2. Aufl.), Bonn. https://www.volkshochschule. de/microsite/grundbildung/unterrichten/Curricula.php.
[30.04.2020].Deutsches Institut für Erwachsenenbildung (Hrsg.) (2019). Curriculum Finanzielle Grundbildung. Bonn: DIE.

Deutsches Institut für Erwachsenenbildung (Hrsg.) (o.J.). Projekt CurVe. Kompetenzmodell "Finanzielle Grundbildung“. https:// www.die-bonn.de/curve/content/pdf/curve-kompetenz modell_finanzielle\%20grundbildung.pdf [29.06.2021].

Deutsches Institut für Erwachsenenbildung (Hrsg.) (2019). Curriculum Finanzielle Grundbildung. Bonn: DIE.

Dinkelaker, J. (2018). Lernen Erwachsener. Stuttgart: Kohlhammer. Europäische Kommission (2018). EMPFEHLUNG DES RATES vom 22. Mai 2018 zu Schlüsselkompetenzen für lebenslanges Lernen. https://eur-lex.europa.eu/legal-content/DE/TXT/PDF/?uri=CEL EX:32018H0604(01)\&from=SV

Evans, J., Yasukawa, K., Mallows, D. \& Creese, B. (2017). Numeracy Skills and the Numerate Environment: Affordances, Opportunities, Supports and Demands. Adults Learning Mathematics, 12(1), $17-26$.

Fritz, A., Haase, V. G. \& Räsänen, P. (2019). International handbook of mathematical learning difficulties. From laboratory to the classroom. Cham: Springer. https ://doi.org/10.1007/978-3319-97148-3.

Gal, I. (2000). The Numeracy Challenge. In I. Gal (Hrsg.), Adult Numeracy Development. Theory, Research, Practice (S.9-31). Cresskill, New Jersey: Hampton Press.

Garrote, A., Moser Opitz, E. \& Ratz, C. (2015). Mathematische Kompetenzen von Schülerinnen und Schülern mit dem Förderschwerpunkt geistige Entwicklung: Eine Querschnittstudie. Empirische Sonderpädagogik, 7(1), 24-40.

Geiger, V., Goose, M. \& Forgasz, H. (2015). A rich interpretation of numeracy for the 21 st century: a survey of the state of the field. ZDM - The International Journal on Mathematics Education, 47(4), 531 - 548. https://doi.org/10.1007/s11858-015-0708-1.

Grassmann, M. (2013). Größenvorstellungen von Geldwerten entwickeln. Mathematik differenziert, 4, 9-11.

Grosche, M., Wohne, A. \& Rüsseler, J. (2016). Zentrale theoretische Ansätze und ihr Einfluss auf unser Denken und Handeln bei funktionalem Analphabetismus. In C. Löffler \& J. Korfkamp (Hrsg.), Handbuch zur Alphabetisierung und Grundbildung Erwachsener (S.129-143). Münster: Waxmann.

Grotlüschen, A. \& Buddeberg, K. (2020). LEO 2018 - Leben mit geringer Literalität. Bielefeld:WBV Media.

Holm, U. (2018). Anthropologische Voraussetzungen des Lernens Erwachsener - Lernfähigkeit als Grundlage der Erwachsenenbildung. In R. Tippel \& A. von Hippel (Hrsg.), Handbuch Erwachsenenbildung/Weiterbildung. 6. überarb. und erw. Aufl. (S.109-125). Wiesbaden: Springer VS.

Klafki, W. (1991). Grundzüge eines neuen Allgemeinbildungskonzepts. Im Zentrum: Epochaltypische Schlüsselprobleme. In W. Klafki (Hrsg.), Neue Studien zur Bildungstheorie und Didaktik. Zeitgemäße Allgemeinbildung und kritisch-konstruktive Didaktik, 2. erw. Aufl. (S.43 - 81). Weinheim und Basel: Beltz Verlag.

Klein, R. \& Alke, M. (2009). Lernberatung und Kompetenzentwicklung: „Ich hatte immer eine Vision im Kopf, wie Lernen stattfinden müsste". In A. Bolder \& R. Dobischat (Hrsg.), Eigen-Sinn und Widerstand (S.243-259). Wiesbaden: VS Verl.

Klieme, E. \& Leutner, D. (2006). Kompetenzmodelle zur Erfassung individueller Lernergebnisse und zur Bilanzierung von Bildungsprozessen. Beschreibung eines neu eingerichteten Schwerpunktprogramms der DFG. Zeitschrift für Pädagogik, 52 (6), 876 - 903.

Koller, J. (2020). Das Grundbildungsverständnis in einer doppelten empirischen Annäherung - Divergenzen, Disziplinen und betriebliche Akteure. In J. Koller, D. Klinkhammer \& M. Schemmann (Hrsg.), Arbeitsorientierte Grundbildung und Alphabetisierung (S.19-42). Bielefeld: WBV.

Konferenz der Kultusminister der Länder in der Bundesrepublik Deutschland (2004). Bildungsstandards im Fach Mathematik 
für den Primarbereich. Beschluss vom 15.10.2004. Luchterhand. https://www.kmk.org/fileadmin/veroeffentlichungen_ beschluesse/2004/2004_10_15-Bildungsstandards-MathePrimar.pdf. [29.06.2021].

Lane, C., Herwegen, J. van \& Freeth, M. (2019). Exploring the approximate number system in Sotos syndrome: insights from a dot comparison task. Journal of Intellectual Disability Research, 63(8), S.917- 925.

Lave, J. (1988). Cognition in practice. Mind, mathematics and culture in everyday life. Cambridge: Cambridge University Press.

Lüssenhop, M. \& Kaiser, G. (2021). Numeralität im Wandel der Zeiten. Analyse zentraler Konzeptionen zu Numeralität und Implikationen für die Numeralitätsdiskussion in der Erwachsenenbildung. Zeitschrift für Pädagogik, 67, Beiheft, S. 36 - 52.

Mangold, W. (1960). Gegenstand und Methode des Gruppendiskussionsverfahrens. Frankfurt a. M.: Europäische Verlagsanstalt.

Mania, E. \& Tröster, M. (2015). Finanzielle Grundbildung. Programme und Angebote planen. Bielefeld: wbv.

Mania, E. \& Tröster, M. (2018). Inhaltsbereiche der Grundbildung. Stand und Herausforderungen. In Agentur für Erwachsenenund Weiterbildung (Hrsg.), Grundbildung lebensnah gestalten. Fallbeispiele aus den Regionalen Grundbildungszentren in Niedersachsen (S.11-21). Bielefeld: wbv.

Mey, G. \& Ruppel, P.S. (2018). Qualitative Forschung. In O. Decker (Hrsg.), Sozialpsychologie und Sozialtheorie, Bd. 1 (S. 205 - 244). Wiesbaden: Springer VS.

Meyerhöfer, W. (2017). Einführung. In DVV Deutscher Volkshochschulverband (Hrsg.), Rechnen. DVV Rahmencurriculum (2. Aufl. S. 3-5). Bonn: DVV.

Niediek, I. (2010). Das Subjekt im Hilfesystem. Eine Studie zur Individuellen Hilfeplanung im Unterstützten Wohnen für Menschen mit einer geistigen Behinderung. Wiesbaden:VS.

Nieß, M. (2016). Partizipation aus Subjektperspektive. Zur Bedeutung von Interessenvertretung für Menschenmit Lernschwierigkeiten. Wiesbaden: Springer.

Organisation for Economic Cooperation and Development (OECD), (2019). The Survey of Adult Skills: Reader's companion (3rd edn). Paris: OECD Publishing. https://doi.org/10.1787/f70238c7-en

Pape, N. (2018). Literalität als milieuspezifische Praxis. Eine qualitative Studie aus einer Habitus- und Milieuperspektive zu Teilnehmenden an Alphabetisierungskursen. Münster, New York: Waxmann.

Porter, J. \& Lacey, P. (2005). Researching learning difficulties. A guide for practitioners. London: Paul Chapman Publishing.

Rammstedt, B. (Hrsg.) (2013). Grundlegende Kompetenzen Erwachsener im internationalen Vergleich: Ergebnisse von PIAAC 2012. Münster: Waxmann.

Ratz, C. \& Wittmann, E. C. (2011). Mathematisches Lernen im Förderschwerpunkt geistige Entwicklung. In C. Ratz (Hg.), Unterricht im Förderschwerpunkt geistige Entwicklung (S.129-152). Oberhausen: Athena.

Reckwitz, A. (2004). Die Kontingenzperspektive der 'Kultur'. Kulturbegriffe, Kulturtheorien und das kulturwissenschaftliche Forschungsprogramm In F. Jaeger \& J. Rüsen, Handbuch der Kulturwissenschaften. Band 3: Themen und Tendenzen (S.1 - 20). Stuttgart, Weimar: Metzler.

Schäfer, H. (2020). Mathematik und geistige Behinderung. Grundlagen für Schule und Unterricht. Stuttgart: Kohlhammer.

Schmitz, G. \& Scharlau, R. (1985). Mathematik als Welterfahrung. Die Erschließung von Raum und Zahl für geistig behinderte Kinder. Bonn-Bad Godesberg: Dürr.

Schuppener, S., Heusner, J. \& Müller, N. A. (2020). Forschungsethische Spannungsfelder in Bezug auf Menschen mit sogenannter geistiger und/oder schwerer Behinderung. Teilhabe, 59(4), $147-152$.

Schreiber-Barsch, S. \& Curdt, W. (2021). Grundbildung und Behinderung: zum Verhältnis von pädagogischem Auftrag, subjekt- gelagerten Fähigkeiten und professionellem Vermittlungshandeln am Beispiel von Numeralität. Zeitschrift für Pädagogik. Beiheft 2021 Dekade für Alphabetisierung und Grundbildung von Erwachsenen, 67, Beiheft 1, $200-224$.

Schreiber-Barsch, S., Curdt, W. \& Gundlach, H. (2020). Whose voices matter? Adults with learning difficulties and the emancipatory potential of numeracy practices. ZDM Mathematics Education Special Issue "Numeracy and vulnerability in (early) adult life", 52(2), $581-592$.

Straka, G. A. \& Macke, G. (2009). Berufliche Kompetenz: Handeln können, wollen und dürfen. BWP, 3,14-17.

Strauss, A. \& Corbin, J. (1996). Grounded Theory: Grundlagen Qualitativer Sozialforschung. Weinheim: Beltz, Psychologie Verlags Union.

Strauss, A. \& Corbin, J. (1990). Basics of qualitative research. Grounded theory procedures and techniques. California: SAGE.

Street, B., Baker, D. \& Tomlin, A. (2008). Navigating numeracies. Home/school numeracy practices. Dordrecht: Springer.

Strübing, J. (2014). Grounded Theory: Zur sozialtheoretischen und epistemologischen Fundierung eines pragmatistischen Forschungsstils. Wiesbaden: Springer.

Tan, P., Lambert, R., Padilla, A. \& Wieman, R. (2019). A disability studies in mathematics education review of intellectual disabilities: Directions for future inquiry and practice. Journal of Mathematical Behavior. https://doi.org/10.1016/j.jmathb.2018.09.001.

Tenorth, H.-E. (2004). Stichwort:,Grundbildung“ und „Basiskompetenzen" Herkunft, Bedeutung und Probleme im Kontext allgemeiner Bildung. Zeitschrift für Erziehungswissenschaft, 7 , $169-182$

Trescher, H. (2018). Kognitive Beeinträchtigung und Barrierefreiheit. Eine Pilotstudie. Bad Heilbrunn: Klinkhardt.

Tröster, M. (2000). Grundbildung - Begriffe, Fakten, Orientierungen. In M. Tröster (Hrsg.), Spannungsfeld Grundbildung (S.12-27). Bielefeld: Bertelsmann.

Tröster, M. \& Schrader, J. (2016). Alphabetisierung, Grundbildung, Literalität: Begriffe, Konzepte, Perspektiven. In C. Löffler \& J. Korfkamp (Hrsg.), Handbuch zur Alphabetisierung und Grundbildung Erwachsener (S. 42 - 58). Münster, u.a.: Waxmann.

von Unger, H. (2014). Partizipative Forschung. Einführung in die Forschungspraxis. Wiesbaden: Springer VS.

Waldschmidt, A. (2017). Disability goes cultural: The cultural model of disability as an analytical tool. In A. Waldschmidt, H. Benessen \& M. Ingwersen (Eds). Culture - theory - disability: Encounters between disability studies and cultural studies (S.19-27). Bielefeld: Transcript.

Weisser, J. (2018). Inklusion, Fähigkeiten und Disability Studies. In T. Sturm \& M. Wagner-Willi (Hrsg.), Handbuch schulische Inklusion (S.93-106). Opladen, Toronto: Barbara Budrich.

Werner, B. (2017). Teilhabe durch Grundbildung. Die Förderung Benachteiligter im Sekundarbereich I. Stuttgart: Kohlhammer.

Williams, V., Tarleton, B., Heslop, P., Porter, S., Sass, B., Blue, S. et al. (2018). Understanding disabling barriers: A fruitful partnership between disability studies and social practices? Disability and Society, 33(2), $157-174$.

Yasukawa, K., Jackson, K., Kane, P. \& Coben, D. (2018). Mapping the terrain of social practice perspectives of numeracy. In K. Yasukawa, A. Rogers, K. Jackson \& B. Street (Hrsg.), Numeracy as social practice. Global and local perspectives (S.3-17). London, New York: Routledge.

Yasukawa, K., Rogers, A., Jackson, K. \& Street, B. (Hrsg.) (2018). Numeracy as social practice. Global and local perspectives. London, New York: Routledge.

Zeuner, C. (2009). Zur Bedeutung gesellschaftlicher Kompetenzen im Sinne eines kritischen bildungstheoretischen Ansatzes. In A. Bolder \& R. Dobischat (Hrsg.), Eigen-Sinn und Widerstand (S. 260 - 281). Wiesbaden: VS Verlag. 
Zeuner, C. \& Pabst, A. (2011). „Lesen und Schreiben eröffnen eine neue Welt!" Literalität als soziale Praxis - eine ethnographische Studie. Bielefeld:W. Bertelsmann.

\section{Historie}

Manuskript eingegangen: 03.03.2021

Manuskript angenommen: 12.08.2021

Onlineveröffentlichung: 31.08.2021

\section{Förderung}

Forschungsprojekt „Numerale Praktiken von Menschen mit Lernschwierigkeiten“ (NumPuD), gefördert durch: Landesforschungsförderung Hamburg (2017 - 2020) (LFF-FV 52).

Open-Access-Veröffentlichung ermöglicht durch die Universität Hamburg.

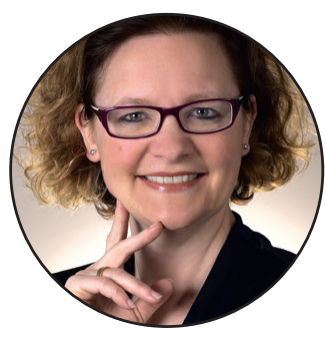

Jun. Prof.in Silke Schreiber-Barsch

Juniorprofessorin für

Erwachsenenbildung

Arbeitsbereich Lebenslanges Lernen

Fakultät für Erziehungswissenschaft

Universität Hamburg

Von Melle Park 8

20146 Hamburg

Deutschland

silke.schreiber-barsch@

uni-hamburg.de 